\title{
Shortened multidrug-resistant tuberculosis regimens overcome low-level fluoroquinolone resistance
}

\author{
To the Editor:
}

We read the research letter by JAVAID et al. [1] with great interest. The recommendations from the World Health Organization (WHO) on the use of shortened multidrug-resistant tuberculosis (MDR-TB) regimens controversially indicated that "shortened MDR-TB regimens should not be used in patients who have documented or likely resistance to medicines in the regimen" [2], leading to the concept that MDR-TB patients with additional resistance to not only fluoroquinolones (FQs) or second-line injectables (SLIs), but also ethambutol (EMB), prothionamide (Pto) and pyrazinamide (PZA) would not be eligible for the regimens [3]. This is a highly conservative approach that will greatly limit the applicability of shortened MDR-TB regimens and deprive many MDR-TB patients in high-burden countries of short and highly effective regimens. The 9-month MDR-TB regimen piloted in Bangladesh comprised high-dose gatifloxacin $\left({ }^{\mathrm{h}} \mathrm{Gfx}\right)$, clofazimine (Cfz), EMB and PZA throughout, supplemented by kanamycin (Km), Pto and high-dose isoniazid $\left({ }^{\mathrm{h}} \mathrm{INH}\right)$ during an intensive phase [4]. The regimen was initially designed to be used in settings with limited resources, where drug susceptibility testing was not available in a timely manner. It was designed as a standardised regimen, using clofazimine and first-line drugs to replace toxic and less effective second-line drugs such as cycloserine and para-aminosalicylic acid. The drugs that are crucial in achieving sputum conversion are ${ }^{\mathrm{h}} \mathrm{Gfx}$ and $\mathrm{Km}$, and those crucial in shortening the duration of treatment are ${ }^{\mathrm{h}} \mathrm{Gfx}, \mathrm{Cfz}$ and PZA [5]. Other drugs, in combination, play a supportive role and full susceptibility to these drugs was not expected in the original design. Most patients treated with shortened MDR-TB regimens in Bangladesh [4] and other countries [6] have been exposed to EMB for a prolonged period. Those with resistance to EMB were not excluded and replacement of EMB by another drug was not performed. ${ }^{\mathrm{h}} \mathrm{INH}$ and Pto were combined complimentarily to address cross-resistance to Pto in inhA-mutant strains of Mycobacterium tuberculosis, since the serum level of ${ }^{\mathrm{h}} \mathrm{INH}$ will exceed the minimum inhibitory concentration (MIC) of strains with inhA mutation by a wide margin, ensuring that at least one of ${ }^{\mathrm{h}} \mathrm{INH}$ and Pto will be effective in most cases. Resistance to Pto was not associated with an unfavourable outcome [4] and ${ }^{\mathrm{h}} \mathrm{INH}$ may also be able to suppress a substantial proportion of bacilli with $k a t G$ mutation.

Shortened MDR-TB regimens using ${ }^{\mathrm{h}} \mathrm{Gfx}$ overcome low-level FQ resistance. In Bangladesh [4], high-level Gfx resistance (MIC $\geqslant 2 \mathrm{mg} \cdot \mathrm{L}^{-1}$ ) but not low-level resistance was significantly associated with bacteriologically unfavourable outcomes (failure or relapse $36.4 \%$ versus $3.2 \%$ ). Another study enrolled a larger number of MDR-TB cases in Bangladesh treated with Gfx, including those analysed by Aung et al. [4] and second-line retreatment cases with a higher prevalence of FQ resistance [7]. With this selection, the proportion of failure/relapse rose from $4 \%$ with Gfx MIC $<1 \mathrm{mg} \cdot \mathrm{L}^{-1}$ to $18 \%, 48 \%$ and $100 \%$ with MIC 1 (low-level), 2-4 (medium) and $>4 \mathrm{mg} \cdot \mathrm{L}^{-1}$ (high), respectively. Table 1 shows the average level of resistance and the proportion of Bangladeshi patients with bacteriologically adverse outcomes by $g y r A / B$ mutation pattern [7].

A considerable proportion of FQ-resistant cases can still reach relapse-free cure with the unmodified short MDR-TB regimen. Using high-dose moxifloxacin (or ${ }^{\mathrm{h}} \mathrm{Gfx}$ ) is crucial to overcome low-level FQ resistance [8]. The poorer outcomes in the second study may be explained by the inclusion of previously second-line treated cases with longstanding selection pressure leading to increased MICs.

@ERSpublications

Shortened unmodified MDR-TB regimens using high-dose gatifloxacin overcome low-level fluoroquinolone resistance http://ow.ly/VCHF30bt4kA

Cite this article as: Van Deun A, Chiang C-Y. Shortened multidrug-resistant tuberculosis regimens overcome low-level fluoroquinolone resistance. Eur Respir J 2017; 49: 1700223 [https://doi.org/10.1183/ 13993003.00223-2017]. 
TABLE 1 Resistance level and impact on treatment outcome of individual gyrA/B mutations

\begin{tabular}{|c|c|c|}
\hline Mutation & FQ resistance level & Failure/relapse outcome \\
\hline gyrA 94 Ala & Low or medium & $11 \%$ \\
\hline gyrA $90 \mathrm{Val}$ & Medium & $47 \%$ \\
\hline $\operatorname{gyrA}^{94 \mathrm{Gly}}$ & High & $70 \%$ \\
\hline gyrA $94 \mathrm{Tyr}$ & High & $100 \%$ \\
\hline gyrA ${ }^{88 C y s}$ & High & Unknown \\
\hline gyrA 89 Asn & High & Unknown \\
\hline gyrA $^{91 \text { Pro }}$ & High & Unknown \\
\hline $\operatorname{gyrA}^{94 \mathrm{Asn} / \mathrm{His}}$ & High & Unknown \\
\hline$g y r B^{538 S e r}$ & High & Unknown \\
\hline gyrB $B^{540 A r g}$ & High & Unknown \\
\hline Multiple mutations & High & Unknown \\
\hline
\end{tabular}

FQ: fluoroquinolone. Reproduced and modified from [7] with permission from the publisher.

Molecular test systems have made drug-susceptibility testing (DST) accessible for most patients. Recent comparisons with phenotypic DST have made it clear that molecular tests may also be more accurate than the gold standard, leading mainly to an underestimated specificity of molecular tests. For the core first- or second-line drugs, rifampicin and FQs, molecular tests have been proposed as an alternative or complementary part of the gold standard $[9,10]$. The WHO has recently recommended the new version 2 of GenoType MTBDRsl lineprobe assay (LPA) (Hain Lifescience GmbH, Nehren, Germany) for detection of resistance to FQs and SLIs. An independent evaluation showed superiority of the new version, with sensitivity for FQ resistance reaching $95 \%$ and specificity, 98\%. The LPA banding pattern allows the identification of the most frequent mutations. For $g y r A$, low- to medium-level resistance is shown by a MUT 1 or MUT3A band, while high-level alerts are the absence of WT1 without MUT1 showing, MUT2, MUT3B, C or D, and absence of WT3 without a MUT band. Both gyrB MUT1 and MUT2 bands indicate high resistance, as do multiple mutations at one or both loci. This might be applied under programme conditions to guide the application of shortened MDR-TB regimens. As long as they remain susceptible to an injectable, effective treatment of cases with low-level resistance to FQs with the shortened regimens thus remains possible. JAVAID et al. [1] pointed out that in Pakistan, this means the large majority of FQ-resistant MDR-TB cases. For cases with medium- or high-level resistance, replacing the FQs with a new drug, such as bedaquiline, is likely the best option.

Armand Van Deun ${ }^{1,2}$ and Chen-Yuan Chiang ${ }^{1,3,4}$

${ }^{1}$ International Union Against Tuberculosis and Lung Disease, Paris, France. ${ }^{2}$ Mycobacteriology Unit, Institute of Tropical Medicine, Antwerp, Belgium. ${ }^{3}$ Division of Pulmonary Medicine, Dept of Internal Medicine, Wan Fang Hospital, Taipei Medical University, Taipei, Taiwan. ${ }^{4}$ Dept of Internal Medicine, School of Medicine, College of Medicine, Taipei Medical University, Taipei, Taiwan.

Correspondence: Chen-Yuan Chiang, Dept of Internal Medicine, School of Medicine, College of Medicine, Taipei Medical University, 111 Hsin-Long Road, Section 3, Taipei, Taiwan. E-mail: cychiang@theunion.org

Received: Jan 312017 | Accepted after revision: Feb 262017

Conflict of interest: None declared

\section{References}

1 Javaid A, Ahmad N, Khan AH, et al. Applicability of the World Health Organization recommended new shorter regimen in a multidrug-resistant tuberculosis high burden country. Eur Respir J 2016; 49: 1601967.

2 Varaine F, Gugliemetti L, Huerga H, et al. Eligibility for the shorter multidrug-resistant tuberculosis regimen: ambiguities in the World Health Organization recommendations. Am J Respir Crit Care Med 2016; 194: 1028-1029.

3 Lange C, Duarte R, Fréchet-Jachym M, et al. Limited benefit of the new shorter multidrug-resistant tuberculosis regimen in Europe. Am J Respir Crit Care Med 2016; 194: 1029-1031.

4 Aung KJM, Van Deun A, Declercq E, et al. Successful '9-month Bangladesh regimen' for multidrug-resistant tuberculosis among over 500 consecutive patients. Int J Tuberc Lung Dis 2014; 18: 1180-1187.

5 Chiang C-Y, Van Deun A, Rieder HL. Gatifloxacin for short, effective treatment of multidrug-resistant tuberculosis. Int J Tuberc Lung Dis 2016; 20: 1143-1147.

6 Piubello A, Harouna SH, Souleymane MB, et al. High cure rate with standardised short-course multidrug-resistant tuberculosis treatment in Niger: no relapses. Int J Tuberc Lung Dis 2014; 18: 1188-1194.

7 Rigouts L, Coeck N, Gumusboga M, et al. Specific gyrA gene mutations predict poor treatment outcome in multidrug-resistant tuberculosis. J Antimicrob Chemother 2016; 71: 314-323. 
8 Gumbo T, Louie A, Deziel MR, et al. Selection of a moxifloxacin dose that suppresses drug resistance in Mycobacterium tuberculosis, by use of an in vitro pharmacodynamic infection model and mathematical modeling. J Infect Dis 2004; 190: 1642-1651.

9 Van Deun A, Aung KJ, Bola V, et al. Rifampin drug resistance tests for tuberculosis: challenging the gold standard. J Clin Microbiol 2013; 51: 2633-2640.

10 Bernard C, Veziris N, Brossier F, et al. Molecular diagnosis of fluoroquinolone resistance in Mycobacterium tuberculosis. Antimicrob Agents Chemother 2015; 59: 1519-1524.

Copyright @ERS 2017 